Subject-specific geometry affects acetabular contact pressure during gait more than subject-specific loading patterns

Mariska Wesseling ${ }^{\mathrm{a}}$, Sam Van Rossom ${ }^{\mathrm{a}}$, Ilse Jonkers ${ }^{\mathrm{a}}$, Corinne R. Henak ${ }^{\mathrm{b} *}$

${ }^{a}$ KU Leuven, Belgium; ${ }^{b}$ University of Wisconsin, Madison, US

Corresponding author:

Corinne R. Henak

Department of Mechanical Engineering

Department of Biomedical Engineering (affiliate)

University of Wisconsin - Madison

3031 Mechanical Engineering Building

1513 University Avenue

Madison, WI 53706

E-mail: chenak@wisc.edu 


\section{Subject-specific geometry affects acetabular contact pressure during gait more than subject-specific loading patterns}

Finite element modeling (FEM) can predict hip cartilage contact mechanics. This study investigated how subject-specific boundary conditions and joint geometry affect acetabular cartilage contact mechanics using a multi-scale workflow. For two healthy subjects, musculoskeletal models calculated subject-specific hip kinematics and loading, which were used as boundary conditions for FEM. Cartilage contact mechanics were predicted using either generic or subject-specific FEM and boundary conditions. A subject-specific mesh resulted in a more lateral contact. Effects of subject-specific boundary conditions varied between both subjects. Results highlight the complex interplay between loading and kinematics and their effect on cartilage contact mechanics.

Keywords: multi-scale modelling, hip contact mechanics, musculoskeletal modelling, finite element modelling 


\section{Introduction}

Osteoarthritis (OA) is the most prevalent joint disease (Zhang \& Jordan 2010). Lifetime risk of symptomatic hip OA is approximately 1 in 4 (Murphy et al. 2010). Hip OA is often secondary to pathomorphologies such as acetabular dysplasia and femoroacetabular impingement (Ganz et al. 2008). Across joints, mechanical loading is important in OA initiation and progression, with cartilage contact pressure serving as an accurate predictor of cartilage degeneration (Carter et al. 2004; Segal et al. 2009).

Finite element modeling (FEM) can predict cartilage contact mechanics. FEM has shown that hip pathomorphologies affect cartilage loading (Yoshida et al. 2006; Russell et al. 2006; Chegini et al. 2009; Henak, Abraham, et al. 2014; Hellwig et al. 2015; Ng et al. 2017). However, in many studies, hip geometry is simplified (e.g. Yoshida et al. 2006; Chegini et al. 2009; Hellwig et al. 2015). This despite hip contact mechanics being sensitive to subjectspecific cartilage and bone geometry (Anderson et al. 2010).

In addition, previous studies have typically employed forces measured using instrumented prostheses as generic boundary conditions. However, gait characteristics are altered in patients with OA and pathomorphology (Mantovani et al. 2012; Skalshøi et al. 2015; Harris et al. 2017; Meyer et al. 2018), thereby altering joint loading patterns (Wesseling et al. 2015; Wesseling et al. 2018). These alterations are likely to affect cartilage contact mechanics. To the best of our knowledge, only one recent study employed subject-specific boundary conditions, calculated using musculoskeletal models, when considering hip cartilage contact mechanics (Ng et al. 2017). Another study used an average dysplastic, but not subject-specific, gait pattern, which altered hip contact stress patterns compared to using a generic gait pattern (Thomas-Aitken et al. 2018).

To determine the effect of altered joint loading on cartilage pressure, musculoskeletal modeling can be combined with FEM, allowing macro- and micro-scale coupling. In such a 
multi-scale workflow, musculoskeletal models and multi-body simulations calculate muscle forces and internal joint loading (Mantovani et al. 2012; Meyer et al. 2018; Wesseling et al. 2018). Calculated joint loading and kinematics then serve as boundary conditions for FEM.

The goal of this study was to investigate how subject-specific boundary conditions and geometry affect acetabular cartilage contact mechanics using a multi-scale modelling workflow. We hypothesized that subject-specific geometry would increase the magnitude and distribute cartilage contact pressure over a smaller area when using subject-specific boundary conditions, consistent with previous research using generic boundary conditions (Anderson et al. 2010). In addition, we hypothesized that subject-specific boundary conditions would increase magnitude and distribute cartilage contact pressures over a larger area, as increased hip contact forces calculated using musculoskeletal modelling compared to instrumented prosthesis have been reported (Mellon et al. 2013; Wesseling, Derikx, et al. 2015). As between healthy and pathomorphologic subjects a difference of about 1-5 MPa in contact pressure and $20 \%$ in contact area was reported (Russell et al. 2006; Chegini et al. 2009; Henak et al. 2013), increases larger than these magnitudes were considered relevant.

\section{Materials and Methods}

\section{Data collection}

Two healthy subjects (S1: female, 26 yrs., 73.4kg, BMI: $24.0 \mathrm{~kg} / \mathrm{m}^{2} ; \mathrm{S} 2:$ male, 26 yrs., $75.1 \mathrm{~kg}$, BMI: $22.4 \mathrm{~kg} / \mathrm{m}^{2}$ ) participated in this study. The study was approved by the ethics committee at UZ Leuven and all subjects signed informed consent. One gait trial at a self-selected walking speed on an $8 \mathrm{~m}$ walkway was used for each subject (S1: $1.28 \mathrm{~m} / \mathrm{s} ; \mathrm{S} 2: 1.39 \mathrm{~m} / \mathrm{s})$. Threedimensional marker trajectories of 65 retroreflective markers were captured using 10 Vicon cameras (100 Hz, Vicon, Oxford Metrics, Oxford, UK). A full body Plug-in-Gait marker set was used (Davis et al. 1991) extended with a three-marker cluster on the upper and lower legs 
and arms and an additional marker on the sacrum and both medial condyles and malleolli. Ground reaction forces were measured using two walkway-embedded force platforms (1000 Hz, Advanced Mechanical Technology Inc., Watertown, MA).

Lower extremity MRIs were collected with subjects lying supine with extended knees. First, a series of four axial T1 weighted SE images were acquired (voxel size $0.93 \times 0.93 \times 1$ mm; 3.0T Philips Ingenia, Philips Healthcare, Best, the Netherlands). A full-leg image was created by combining overlapping image series. To allow coupling of kinematic and MRI data, radio opaque, non-ferromagnetic markers were placed prior to imaging on outlined marker positions of the reflective gait analysis markers (Scheys et al. 2006). Second, 3-dimensional gradient echo water-selective/fluid (3D WATSf) sequence MR images were acquired to visualize cartilage (voxel size $0.29 \times 0.29 \times 0.86 \mathrm{~mm}$ ). To widen the joint space and visualize both cartilage layers, $15 \mathrm{~kg}$ of traction was applied (Abraham et al. 2015; Mechlenburg et al. 2015). Acquisition time of the 3D WATSf scan was $5 \mathrm{~min} 49 \mathrm{sec}$ and traction was applied at least 2 min before imaging.

\section{Musculoskeletal modelling}

Musculoskeletal MRI-based models were created for both subjects using a previously established and validated workflow (Scheys et al. 2006). Briefly, pelvis, femora, patellae and tibiae were segmented (Mimics Innovation Suite, Materialise N.V., Leuven, Belgium). Hip joint center and knee axis were defined based on femoral head and condyle geometries, respectively. Segmental coordinate frames were defined (Wu et al. 2002). Model marker coordinates from radio opaque markers, to be used in dynamic simulations of motion, were expressed in respective segmental coordinate frames. Next, muscle points of all hip and knee actuating muscles were identified in MR images (Scheys et al. 2006). Wrapping surfaces were included to simulate the more complex muscle path of hip flexor muscles (Wesseling et al. 2016). Muscle points of all distal tibia and foot muscles as well as the ankle joint center were 
adopted from a generic model (Delp et al. 1990), which was scaled based on a static trial (Software for Interactive Musculoskeletal Modeling (SIMM), Motion Analysis Corp., Santa Rosa, CA, US).

For the subject-specific loading condition, dynamic simulations of gait were generated in OpenSim 3.3 (Delp et al. 2007). Joint angles during gait were calculated using Kalman smoothing (De Groote et al. 2008), where model markers were fitted to experimental marker trajectories with a least squares optimization. Static optimization was used to estimate muscle forces, while minimizing the sum of squared muscle activations (Anderson \& Pandy 2001). Hip contact forces, i.e. the joint forces between the femur and pelvis as a result of all loads (including muscle forces) acting on the joint, were calculated using the JointReaction analysis in OpenSim.

For the generic loading condition, hip contact forces and kinematics from a patient with an instrumented hip prosthesis were used (Bergmann et al. 2001), similar to previous research (e.g. Yoshida et al. 2006; Chegini et al. 2009; Hellwig et al. 2015). Trial HSRWN6 from the HIP98 database was selected, as this subject showed the typical double peak pattern in hip contact force.

\section{Finite element modelling}

Subject-specific FEM geometry was created based on 3D WATSf MRI scans. Pelvic and femoral bones and cartilage layers were semi-automatically segmented from MR images (Mimics Innovation Suite, Materialise N.V., Leuven, Belgium). Cartilage was discretized into quadratic tetrahedral elements (S1: 73,879 and 20,690 elements; average element volume of 0.122 and $0.189 \mathrm{~mm}^{3}, \mathrm{~S} 2: 35,103$ and 18,551 elements; average element volume of 0.295 and $0.319 \mathrm{~mm}^{3}$ for femoral and acetabular cartilage, respectively) and represented as nearlyincompressible Neo-Hookean material (shear modulus $=13.6 \mathrm{MPa}$, bulk modulus $=1359 \mathrm{MPa}$ ). 
Incompressibility was confirmed by checking the volume ratio, which was below $4 \%$ for all simulations. Bones were represented as rigid bodies.

For comparison, a publically available hip FEM was used as generic geometry (hip_n10rb from the FEBio Example Problems; http://febio.org). In this model, cartilage was discretized into hexahedral elements (24,300 and 15,000 elements; average element volume of 0.203 and $0.179 \mathrm{~mm}^{3}$ for femoral and acetabular cartilage, respectively) with the same nearlyincompressible Neo-Hookean material properties (shear modulus $=13.6 \mathrm{MPa}$, bulk modulus $=$ $1359 \mathrm{MPa}$, confirmed by checking volume ratio) and rigid bones. Although cartilage was discretized into different element types for subject-specific and generic geometries, the similar number of elements allows comparison (Maas et al. 2016). Therefore, mesh density of subjectspecific meshes was at least that of the generic mesh and no convergence study was performed.

Cartilage contact pressures were calculated using FEBio 2.6 (Maas et al. 2012) for three different model setups: generic FEM with subject-specific hip contact forces and joint rotations (generic mesh); subject-specific FEM with generic hip contact forces and joint rotations (generic loading); and subject-specific FEM with subject-specific hip contact forces and joint rotations (subject-specific). This allowed us to test two hypotheses: that subject-specific geometry (generic mesh vs. subject-specific) and subject-specific loading patterns (generic loading vs. subject-specific) affect magnitude and distribution of cartilage contact pressures.

\section{Data processing}

Acetabular cartilage was divided into six approximately equally-sized anatomical regions (figure 1): anterolateral (AL), superolateral (SL), posterolateral (PL), anteromedial (AM), superomedial (SM) and posteromedial (PM) (Athanasiou et al. 1994). Contact area, calculated as percentage of faces in contact, and median contact pressure over contacting faces, with $25^{\text {th }}$ and $75^{\text {th }}$ percentile, were calculated for full cartilage and for all anatomical regions. Pressure 
maps were presented at five instances of the gait cycle (heel contact, instant of first peak in hip contact force, minimum hip contact force during mid-stance, second peak in hip contact force and toe off). Between healthy and pathomorphologic subjects, a difference of about 1-5 MPa in contact pressure and 20\% in contact area was reported (Russell et al. 2006; Chegini et al. 2009; Henak et al. 2013). Therefore, a difference of more than $5 \mathrm{MPa}$ in contact pressure and $20 \%$ in contact area was considered relevant.

To quantify differences in model geometries, cartilage geometries were compared by evaluating cartilage thickness and by fitting a conchoid to the acetabular cartilage surface (Preview 1.20.4, https://febio.org/preview/) (Cerveri et al. 2014). To determine the fit of the conchoid to the acetabular surface, fitting errors (indicating the level of conchoids-shape of the acetabulum) and radii $\mathrm{a}$ and $\mathrm{b}$ (indicating the size) of the conchoid equation $(\mathrm{r}=\mathrm{a}+\mathrm{b} \cos (\theta))$ are reported (Anderson et al. 2010).

\section{Results}

\section{Kinematics and kinetics}

Generic hip kinematics were mostly different from S1 and S2 in the frontal plane, showing increased hip adduction in the second half of stance phase (maximal difference with generic $\mathrm{S} 1: 13.5^{\circ}, \mathrm{S} 2: 18.7^{\circ}$; figure 2 , top). At the end of stance phase, generic hip flexion was slightly increased compared to both subjects (maximal difference with generic S1: $11.4^{\circ}, \mathrm{S} 2: 14.5^{\circ}$ ). Generic vertical hip contact forces were decreased compared to subject-specific loading (maximal difference with generic S1: $3.90 \mathrm{BW}, \mathrm{S} 2: 2.47 \mathrm{BW}$; figure 2, bottom). In addition, lateral (maximal difference with generic S1:0.65 BW, S2: $0.46 \mathrm{BW}$ ) and anterior (maximal difference with generic S1: 0.99 BW, S2: 1.10 BW) loading components were larger for generic compared to subject-specific loading patterns. 


\section{Joint geometry}

Generic cartilage was slightly thinner compared to that of S1 and S2 (figure 3). Conchoid fits showed generic cartilage was slightly smaller $\left(a=22.3 \mathrm{~mm}, \mathrm{~b}=2.5 \times 10^{-5} \mathrm{~mm}\right)$ than both subjectspecific geometries $\left(\mathrm{S} 1: \mathrm{a}=24.1 \mathrm{~mm}, \mathrm{~b}=-2.1 \times 10^{-5} \mathrm{~mm} ; \mathrm{S} 2: \mathrm{a}=27.8 \mathrm{~mm}, \mathrm{~b}=-2.4 \times 10^{-6} \mathrm{~mm}\right)$. Generic geometry was also more conchoid-shaped, as fitting error was smaller compared to subject-specific geometries (generic: $0.05 \mathrm{~mm}$; S1: $0.25 \mathrm{~mm}$; S2: $0.34 \mathrm{~mm}$ ).

\section{Joint contact mechanics}

Median contact pressures over the contact surfaces were similar between models (maximal difference of medians generic mesh and subject-specific S1: 3.29 MPa, S2: $3.64 \mathrm{MPa}$; generic loading and subject-specific S1: 1.45 MPa, S2: 1.89 MPa; figure 4). For S2, there was a tendency of an increased pressure when using a generic mesh, although differences were limited. Larger differences were apparent in contact area (maximal difference of medians generic mesh and subject-specific S1: $7.95 \%, \mathrm{~S} 2: 22.2 \%$; generic loading and subject-specific $\mathrm{S} 1: 24.0 \%, \mathrm{~S} 2: 11.3 \%$; figure 5), where for S1 generic loading resulted in a decreased contact area at the end of the stance phase. For S2, a generic mesh resulted in an increased contact area.

Distinct regional variations in contact pressures were present as a result of geometry (comparing generic mesh vs. subject-specific; figures 6-8). Using the subject-specific mesh, contact pressure was located more laterally over the entire stance phase, specifically in S2 (figure 6). This decreased contact pressure on AM, SM, and PM acetabular cartilage for the subject-specific model (maximal difference of medians generic mesh and subject-specific AM: S1: 4.20 MPa, S2: 8.19 MPa, SM: S1: 4.88 MPa, S2: 4.80 MPa, PM: S1: $6.37 \mathrm{MPa}, \mathrm{S} 2: 7.24$ MPa; figure 7). The generic mesh increased contact area in the SM region for both subjects (maximal difference of medians generic mesh and subject-specific SM: S1: 71.0\%, S2: 67.2\%; figure 8). In S1, contact area was small in the AL region using the generic mesh (below $3 \%$ in 
the second half of the stance phase), while contact area in S2 was small in the SL and PL regions compared to subject-specific geometry (maximal difference of medians generic mesh and subject-specific: AL: S1: 72.3\%, S2: 38.1\%, SL: S1: 44.3\%, S2: 40.3\%, PL: S1 37.5\%, S2: $22.7 \%)$

Subject-specific loading patterns (comparing generic loading vs. subject-specific) resulted in less distinct differences than subject-specific geometry. For S1, contact pressure was slightly decreased, while for S2, the pressure was slightly increased when using subject-specific compared to generic loading pattern (figure 4). For S2 the difference was most distinct in the AM region, the cartilage was unloaded after $32 \%$ of stance phase for generic loading pattern, while this region was in contact with subject-specific loading (maximal difference of medians generic loading and subject-specific: AM: S1: 3.23 MPa, S2: 4.88 MPa; figure 7). Contact area was increased for S1 when using subject-specific loading pattern, specifically at the end of stance phase on all regions of the cartilage (figure 8). For S2, contact area was slightly increased anteriorly and superiorly (specifically AL and SL) using subject-specific loading, while posteriorly contact area (PL and PM) was slightly decreased (maximal difference of medians generic loading and subject-specific: AL: S1: 31.0\%, S2: 37.5\%, SL: S1: 35.9\%, S2: 21.2\%, PL: S1: 20.1\%, S2: 22.8\%, PM: S1: 24.7\%, S2: 10.4\%).

\section{Discussion}

This study demonstrated that both joint geometry and boundary conditions affect acetabular cartilage contact pressure, confirming our hypotheses. Effects of subject-specific geometry are consistent with a previous study (Anderson et al. 2010). Geometry resulted in differences in median contact pressure up to $8.19 \mathrm{MPa}$ and median contact area up to $72 \%$ (figures 7 and 8), exceeding differences of 1-5 MPa contact pressure and 20\% contact area between healthy and pathomorphologic subjects (Russell et al. 2006; Chegini et al. 2009; Henak et al. 2013). In 
addition, subject-specific boundary conditions resulted in larger magnitude loads and thereby altered contact mechanics. In S1, increased force was distributed over a greater contact area, while in S2, increased force resulted in increased contact pressure. However, the effect of using subject-specific boundary conditions was smaller than the effect of subject-specific geometry. Boundary conditions resulted in differences in median contact pressure up to 4.88 $\mathrm{MPa}$ and median contact area up to $37.5 \%$ (figures 7 and 8), close to differences between healthy and pathomorphologic hips. However, for all configurations contact pressure was increased compared to what was reported for healthy subjects by Harris et al. (2012), who reported an average pressure of about $1 \mathrm{MPa}$ and peak pressures around $7 \mathrm{MPa}$.

Contact pressures varied more in individual anatomical regions (figure 7) than across the whole cartilage (figure 4), indicating a redistribution of loading in different models. Subjectspecific contact geometry resulted in more lateral contact than generic geometry (figures 6 and 7). This indicates that it is important to consider cartilage regions individually. This shift may be important because a shift in loading location can result in cartilage damage (Andriacchi et al. 2004; Van de Velde et al. 2009). Although subject-specific boundary conditions resulted in less pronounced changes in cartilage contact, its effects varied by subject, suggesting its importance in subject-specific modelling.

Results suggest interactions between geometry and boundary conditions (figures 7 and 8). The effect of geometry on contact pressure and area was apparent over the entire stance phase. However, the effect of boundary conditions was more distinct in the second half of stance phase, likely because the generic abduction angle was most different from subject-specific angles in both subjects in this portion of stance. In addition, despite the posterior force being larger for subject-specific than for generic contact forces, this did not result in more posteriorly located contact pressure. Conversely, contact area for S2 was slightly decreased posteriorly when using subject-specific loading, although values were similar or less than what is 
considered clinically relevant (maximal difference of medians generic loading and subjectspecific: PL: 22.8\%, PM: 10.4\%). Increased subject-specific loading did not necessarily result in increased contact pressure. Higher subject-specific loading in S1 increased contact area, while in S2 it increased contact pressure. Although the importance of taking the aberrant gait pattern of dysplasia patients into account when considering contact mechanics has already been reported (Thomas-Aitken et al. 2018), the present study indicates that individual gait pattern of healthy subjects is also important. Together, these results highlight complex interplay between geometry, loading and kinematics and their effect on contact mechanics.

There are several limitations that need to be considered. The musculoskeletal model accounted for subject-specific muscle paths and joint geometries, but muscle-tendon parameters (maximal isometric muscle force, tendon slack length and optimal fiber length) and lower tibia and foot muscles were adopted from the generic model. In the FEM, bones were modelled as rigid bodies, although that can affect cartilage contact mechanics (Anderson et al. 2008). Cartilage was represented as nearly-incompressible Neo-Hookean material, a simplification of articular cartilage behavior. However, this simplification is sufficient to predict contact area and patterns (Henak, Kapron, et al. 2014). No convergence study was conducted, but the number of elements was based on Maas et al. (2016). In this study, they reported similar results for simulations of hip cartilage contact mechanics when using hexahedral or tetrahedral meshes when using a similar number of nodes. Therefore, we expect that the number of elements we used in the present study is sufficient for convergence of the results. The labrum was omitted in these models because previous research showed that the labrum plays a limited role in load transfer in healthy hips (Henak et al. 2011). However, the labrum may be more important when including subject-specific boundary conditions. In this study, FEM was used to predict cartilage contact mechanics, which can be predicted more efficiently using discrete element analysis (Abraham et al. 2013). Because cartilage damage criteria include metrics beyond cartilage 
contact mechanics (e.g., shear stress) that do require FEM to predict, this study employed FEM for future flexibility. Finally, the small numbers in this study precluded statistical tests and only trends could be described.

In conclusion, based on a multi-scale modelling workflow, both joint geometry and boundary condition affect acetabular cartilage contact pressure, with the largest effect from geometry. This suggests the need for both subject-specific contact geometry and loading patterns when considering cartilage mechanics and the resulting risk for cartilage degeneration.

\section{Acknowledgements}

This work was supported by funding of the KU Leuven research council under Grant OT/13/083; and FWO travel grant under Grant V441017N. The funding sources had no involvement in the study.

\section{Declaration of interest}

None of the authors have no potential conflict of interest.

\section{References}

Abraham CL, Bangerter NK, McGavin LS, Peters CL, Drew AJ, Hanrahan CJ, Anderson AE. 2015. Accuracy of 3D dual echo steady state (DESS) MR arthrography to quantify acetabular cartilage thickness. J Magn Reson Imaging. 42:1329-1338.

Abraham CL, Maas SA, Weiss JA, Ellis BJ, Peters CL, Anderson AE. 2013. A new discrete element analysis method for predicting hip joint contact stresses. J Biomech. 46:1121-1127.

Anderson AE, Ellis BJ, Maas SA, Peters CL, Weiss JA. 2008. Validation of Finite Element Predictions of Cartilage Contact Pressure in the Human Hip Joint. J Biomech Eng. 130:1-10.

Anderson AE, Ellis BJ, Maas SA, Weiss JA. 2010. Effects of idealized joint geometry on finite element predictions of cartilage contact stresses in the hip. J Biomech. 43:1351-1357.

Anderson FC, Pandy MG. 2001. Static and dynamic optimization solutions for gait are practically equivalent. J Biomech. 34:153-161.

Andriacchi T, Mündermann A, Smith LR, Alexander EJ, Dyrby CO, Koo S. 2004. A 
framework for the in vivo pathomechanics of osteoarthritis at the knee. Ann Biomed Eng. $32: 447-457$.

Athanasiou KA, Agarwal A, Dzida FJ. 1994. Comparative Study of the Intrinsic Mechanical Properties of the Human Acetabular and Femoral Head Cartilage. J Orthop Res. 12:340-349.

Bergmann G, Deuretzbacher G, Heller M, Graichen F, Rohlmann A, Strauss J, Duda GN. 2001. Hip contact forces and gait patterns from routine activities. J Biomech. 34:859-871.

Carter DR, Beaupre GS, Wong M, Smith RL, Andriacchi TP, Schurman DJ. 2004. The Mechanobiology of Articular Cartilage Development and Degeneration. Clin Orthop Relat Res. 427:S69-S77.

Cerveri P, Manzotti A, Baroni G. 2014. Patient-specific acetabular shape modelling: comparison among sphere, ellipsoid and conchoid parameterisations. Comput Methods Biomech Biomed Engin. 17:560-567.

Chegini S, Beck M, Ferguson SJ. 2009. The effects of impingement and dysplasia on stress distributions in the hip joint during sitting and walking: A finite element analysis. J Orthop Res. 27:195-201.

Davis RB, Ounpuu S, Tyburski D, Gage JR. 1991. A gait analysis data collection and reduction technique. Hum Mov Sci. 10:575-587.

Delp SL, Anderson FC, Arnold AS, Loan P, Habib A, John CT, Guendelman E, Thelen DG. 2007. OpenSim: Open source to create and analyze dynamic simulations of movement. IEEE Trans Biomed Eng. 54:1940-1950.

Delp SL, Loan JP, Hoy MG, Zajac FE, Topp EL, Rosen JM. 1990. An interactive graphicsbased model of the lower extremity to study orthopaedic surgical procedures. IEEE Trans Biomed Eng. 37:757-767.

Ganz R, Leunig M, Leunig-Ganz K, Harris WH. 2008. The etiology of osteoarthritis of the hip: An integrated mechanical concept. Clin Orthop Relat Res. 466:264-272.

De Groote F, De Laet T, Jonkers I, De Schutter J. 2008. Kalman smoothing improves the estimation of joint kinematics and kinetics in marker-based human gait analysis. J Biomech. 41:3390-3398.

Harris MD, Anderson AE, Henak CR, Ellis BJ, Peters CL, Weiss JA. 2012. Finite element prediction of cartilage contact stresses in normal human hips. J Orthop Res. 30:1133-1139.

Harris MD, MacWilliams BA, Bo Foreman K, Peters CL, Weiss JA, Anderson AE. 2017. Higher medially-directed joint reaction forces are a characteristic of dysplastic hips: A comparative study using subject-specific musculoskeletal models. J Biomech. 54:80-87.

Hellwig FL, Tong J, Hussell JG. 2015. Hip joint degeneration due to cam impingement: a finite element analysis. Comput Methods Biomech Biomed Engin.:1-8.

Henak CR, Abraham CL, Anderson AE, Maas SA, Ellis BJ, Peters CL, Weiss JA. 2014. Patient-specific analysis of cartilage and labrum mechanics in human hips with acetabular dysplasia. Osteoarthr Cartil. 22:210-217. 
Henak CR, Carruth ED, Anderson AE, Harris MD, Ellis BJ, Peters CL, Weiss JA. 2013. Finite element predictions of cartilage contact mechanics in hips with retroverted acetabula. Osteoarthritis Cartilage. 21:1522-1529.

Henak CR, Ellis BJ, Harris MD, Anderson AE, Peters CL, Weiss JA. 2011. Role of the acetabular labrum in load support across the hip joint. J Biomech. 44:2201-2206.

Henak CR, Kapron AL, Anderson AE, Ellis BJ, Maas SA, Weiss JA. 2014. Specimen-specific predictions of contact stress under physiological loading in the human hip: validation and sensitivity studies. Biomech Model Mechanobiol. 13:387-400.

Maas SA, Ellis BJ, Ateshian GA, Weiss JA. 2012. FEBio: Finite Elements for Biomechanics. J Biomech Eng. 134:011005.

Maas SA, Ellis BJ, Rawlins DS, Weiss JA. 2016. Finite element simulation of articular contact mechanics with quadratic tetrahedral elements. J Biomech. 49:659-667.

Mantovani G, Lamontagne M, Varin D, Cerulli GG, Beaulé PE. 2012. Comparison of total hip arthroplasty surgical approaches by Principal Component Analysis. J Biomech. 45:2109_ 2115.

Mechlenburg I, Nyengaard JR, Gelineck J, Soballe K. 2015. Cartilage Thickness and Cyst Volume Are Unchanged 10 Years After Periacetabular Osteotomy in Patients Without Hip Symptoms. Clin Orthop Relat Res. 473:2644-2649.

Meyer CAG, Wesseling M, Corten K, Nieuwenhuys A, Monari D, Simon J-P, Jonkers I, Desloovere K. 2018. Hip movement pathomechanics of patients with hip osteoarthritis aim at reducing hip joint loading on the osteoarthritic side. Gait Posture. 59:11-17.

Murphy LB, Helmick CG, Schwartz TA, Renner JB, Tudor G, Koch GG, Dragomir AD, Kalsbeek WD, Luta G, Jordan JM. 2010. One in four people may develop symptomatic hip osteoarthritis in his or her lifetime. Osteoarthr Cartil. 18:1372-1379.

Ng KCG, Mantovani G, Lamontagne M, Labrosse MR, Beaulé PE. 2017. Increased Hip Stresses Resulting From a Cam Deformity and Decreased Femoral Neck-Shaft Angle During Level Walking. Clin Orthop Relat Res. 475:998-1008.

Russell ME, Shivanna KH, Grosland NM, Pedersen DR. 2006. Cartilage contact pressure elevations in dysplastic hips: a chronic overload model. J Orthop Surg Res. 1:6.

Scheys L, Jonkers I, Loeckx D, Maes F. 2006. Image based musculoskeletal modeling allows personalized biomechanical analysis of gait. In: Lect Notes Comput Sci. [place unknown]; p. 58-66.

Segal NA, Anderson DD, Iyer KS, Baker J, James C, Lynch JA, Felson DT, Lewis CE, Brown TD. 2009. Baseline articular contact stress levels predict incident symptomatic knee osteoarthritis development in the MOST cohort. J Orthop Res. 27:1562-1568.

Skalshøi O, Iversen CH, Nielsen DB, Jacobsen J, Mechlenburg I, Søballe K, Sørensen H. 2015. Walking patterns and hip contact forces in patients with hip dysplasia. Gait Posture. 42:529-533. 
Thomas-Aitken HD, Willey MC, Goetz JE. 2018. Joint contact stresses calculated for acetabular dysplasia patients using discrete element analysis are significantly influenced by the applied gait pattern. J Biomech. 79:45-53.

Van de Velde SK, Bingham JT, Hosseini A, Kozanek M, DeFrate LE, Gill TJ, Li G. 2009. Increased tibiofemoral cartilage contact deformation in patients with anterior cruciate ligament deficiency. Arthritis Rheum. 60:3693-3702.

Wesseling M, De Groote F, Bosmans L, Bartels W, Meyer C, Desloovere K, Jonkers I. 2016. Subject-specific geometrical detail rather than cost function formulation affects hip loading calculation. Comput Methods Biomech Biomed Engin. 19:1475-1488.

Wesseling M, De Groote F, Meyer C, Corten K, Simon J-P, Desloovere K, Jonkers I. 2015. Gait alterations to effectively reduce hip contact forces. J Orthop Res. 33:1094-1102.

Wesseling M, Meyer C, Corten K, Desloovere K, Jonkers I. 2018. Longitudinal joint loading in patients before and up to one year after unilateral total hip arthroplasty. Gait Posture. 61:117-124.

Wu G, Siegler S, Allard P, Kirtley C, Leardini A, Rosenbaum D, Whittle M, D'Lima DD, Cristofolini L, Witte H, et al. 2002. ISB recommendation on definitions of joint coordinate system of various joints for the reporting of human joint motion - part I: ankle, hip, and spine. J Biomech. 35:543-548.

Yoshida H, Faust A, Wilckens J, Kitagawa M, Fetto J, Chao EYS. 2006. Three-dimensional dynamic hip contact area and pressure distribution during activities of daily living. J Biomech. 39:1996-2004.

Zhang Y, Jordan JM. 2010. Epidemiology of osteoarthritis. Clin Geriatr Med. 26:355-369. 


\section{Figures}

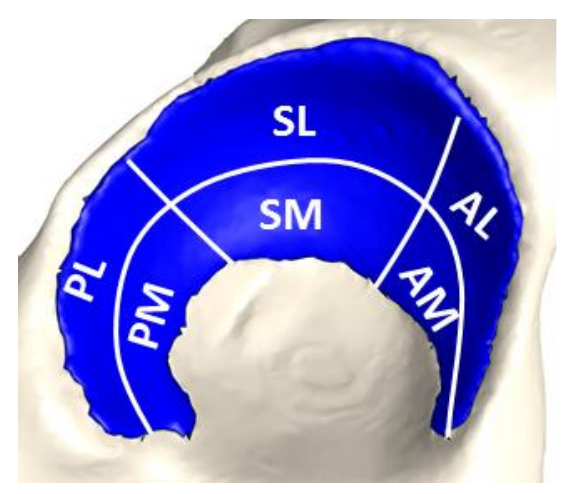

Figure 1. The six anatomical regions on the acetabular cartilage $(\mathrm{AL}=$ anterolateral, $\mathrm{SL}=$ superolateral, $\mathrm{PL}=$ posterolateral, $\mathrm{AM}=$ anteromedial, $\mathrm{SM}=$ superomedial, $\mathrm{PM}=$ posteromedial). 

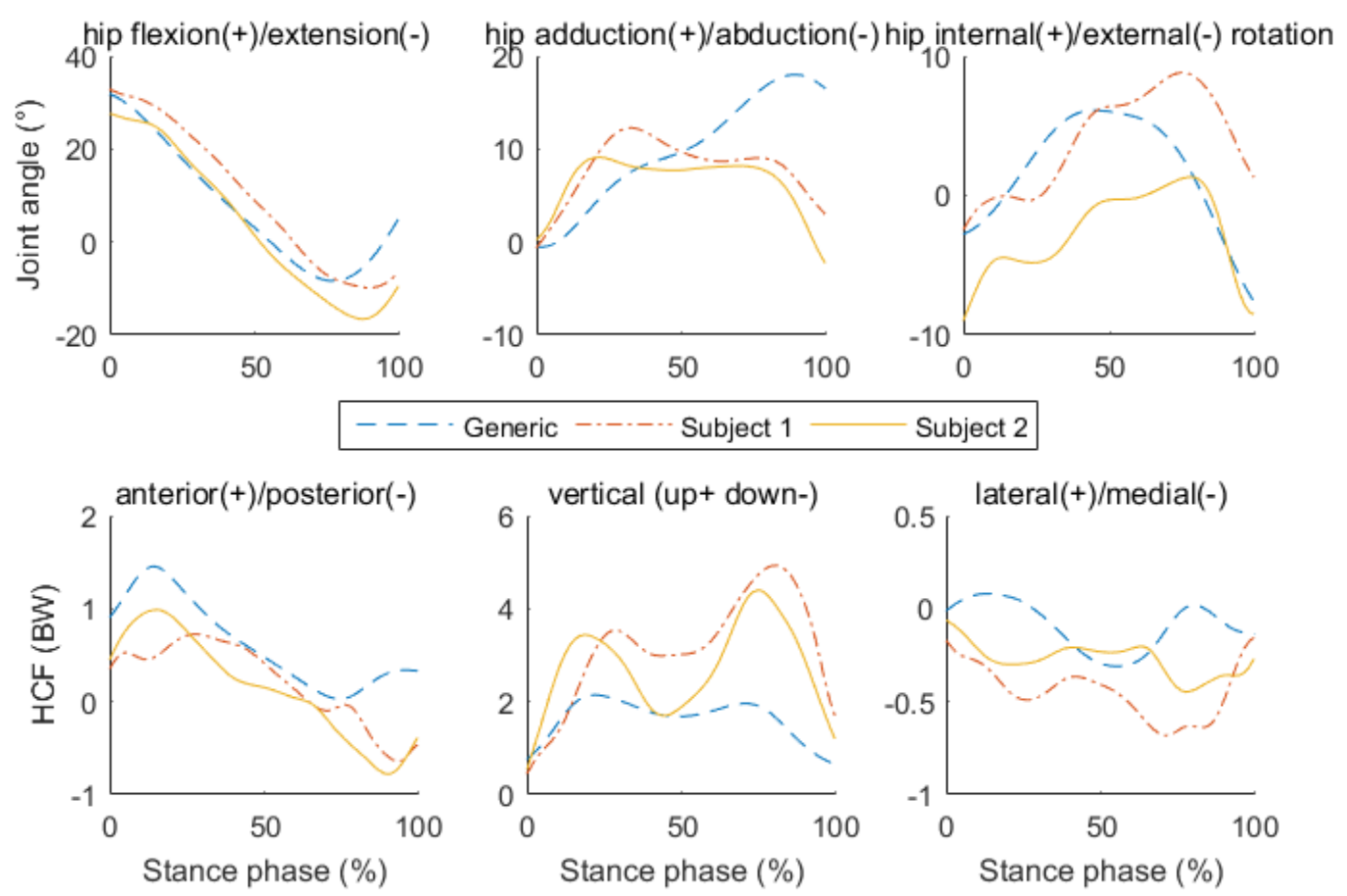

Figure 2. Hip kinematics (top) and contact force (HCF, bottom) during the stance phase of gait for the generic loading pattern (blue) and the subject-specific loading calculated with the musculoskeletal model for subject 1 (red) and 2 (yellow). 


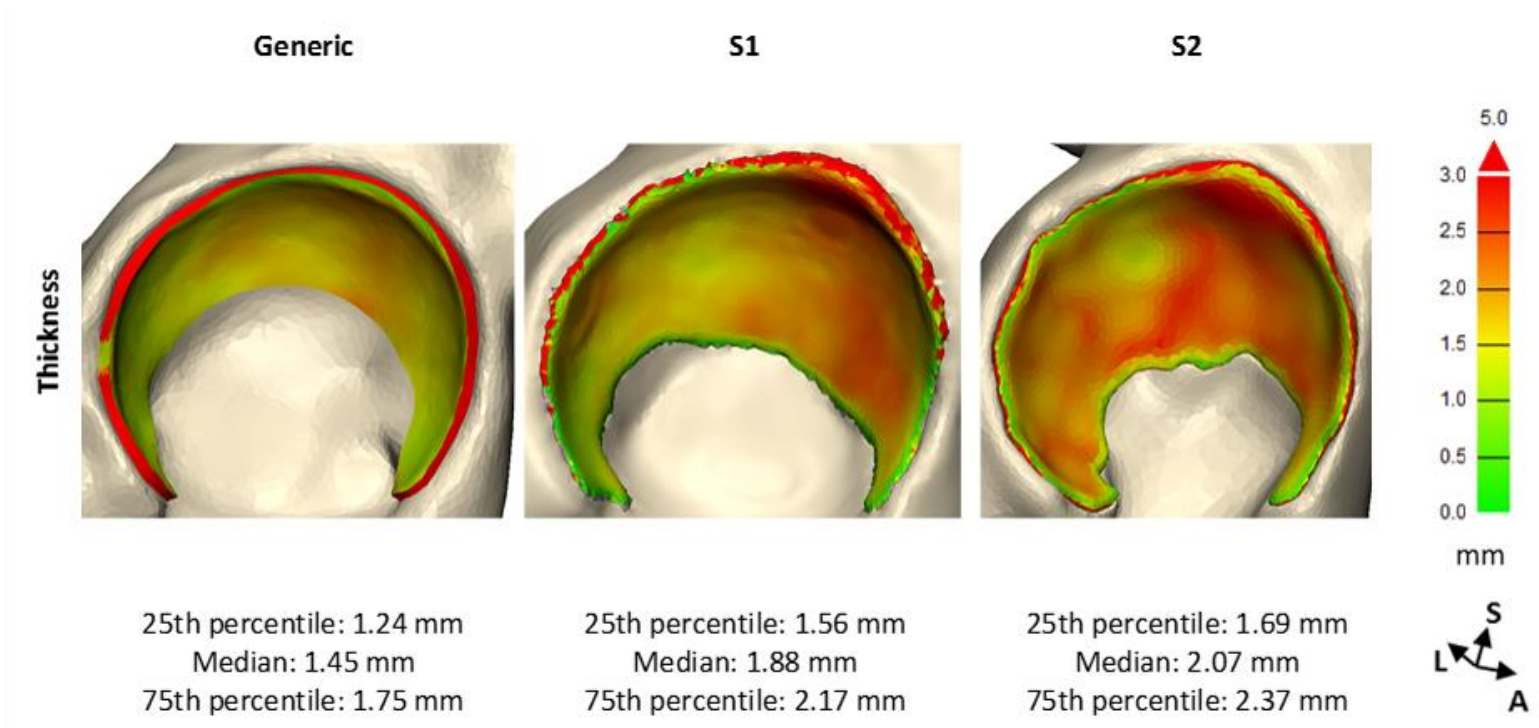

Figure 3. Cartilage thickness of the generic (left), S1 (middle) and S2 (right) acetabular cartilages. The bottom right corner shows the orientation of the anatomical axes $(\mathrm{S}=$ superior; $\mathrm{A}=$ anterior; $\mathrm{L}=$ lateral). 

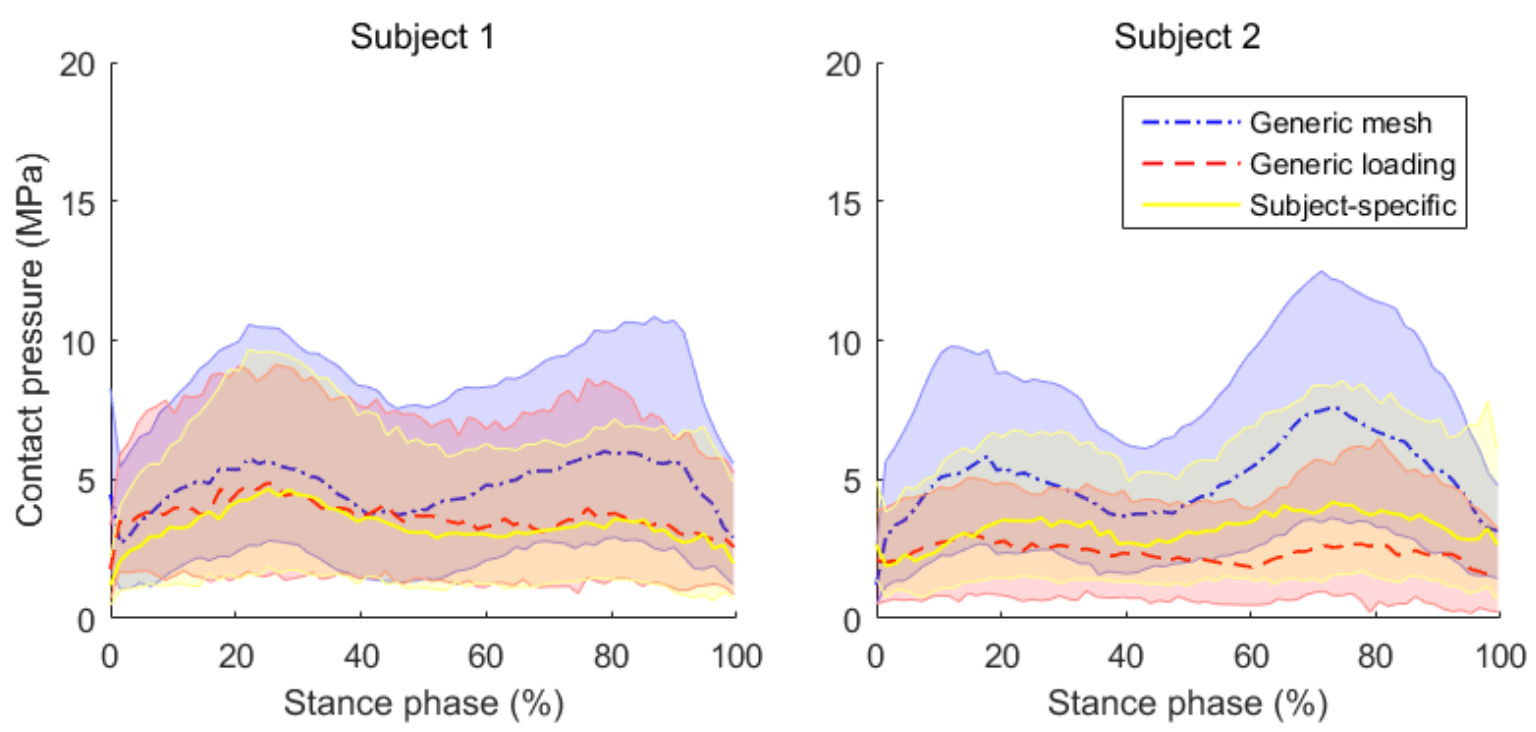

Figure 4. Median contact pressure (and $25^{\text {th }}$ and $75^{\text {th }}$ percentile) on the acetabular cartilage over the stance phase for subject 1 (left) and 2 (right) using the subject-specific mesh with subjectspecific loading (yellow full line), the subject-specific mesh with generic loading (red dashed line) and the generic mesh with subject-specific loading (blue dot-dashed line). 

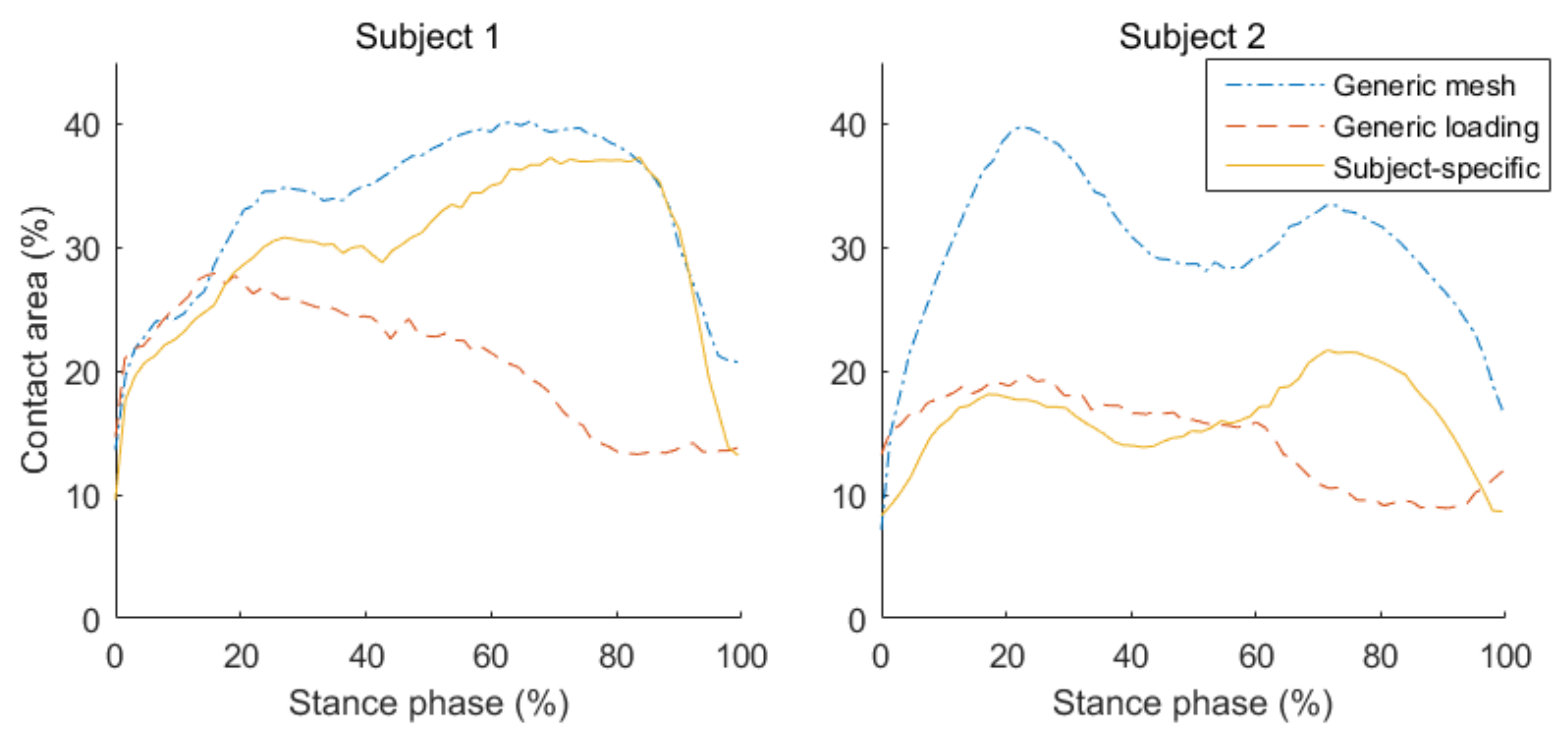

Figure 5. Contact area on the acetabular cartilage over the stance phase for subject 1 (left) and 2 (right) using the subject-specific mesh with subject-specific loading (yellow full line), the subject-specific mesh with generic loading (red dashed line) and the generic mesh with subjectspecific loading (blue dot-dashed line). 


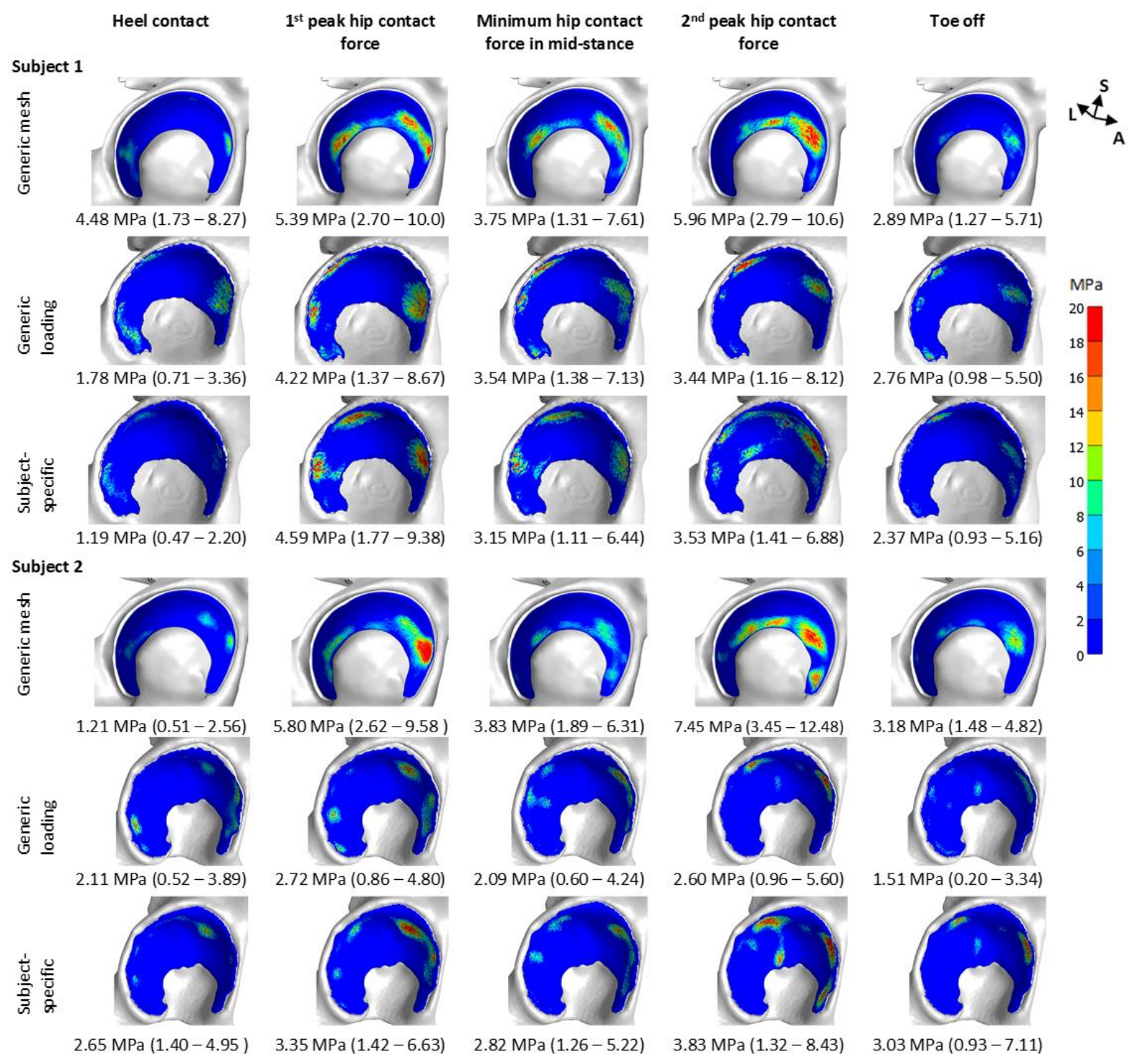

Figure 6. Contact pressure with median (interquartile range) at five time instances of the gait cycle using generic mesh with subject-specific loading, subject-specific mesh with generic loading and subject-specific mesh with subject-specific loading. Heel contact and toe off represent the first and last time instant of stance phase, respectively. The first peak in hip contact force occurs at $20 \%, 30 \%$ and $19 \%$ of the stance phase for generic loading, S1 and S2 respectively. The minimum hip contact force during mid-stance occurs at $54 \%, 48 \%$ and $46 \%$ of stance phase for generic loading, S1 and S2 respectively. The second peak hip contact force occurs at $73 \%, 83 \%$ and $72 \%$ of stance phase for generic loading, S1 and S2 respectively. See 
figure 2 for hip contact force at these times. The top right corner shows the orientation of anatomical axes $(\mathrm{S}=$ superior; $\mathrm{A}=$ anterior; $\mathrm{L}=$ lateral $)$. 
Subject 1
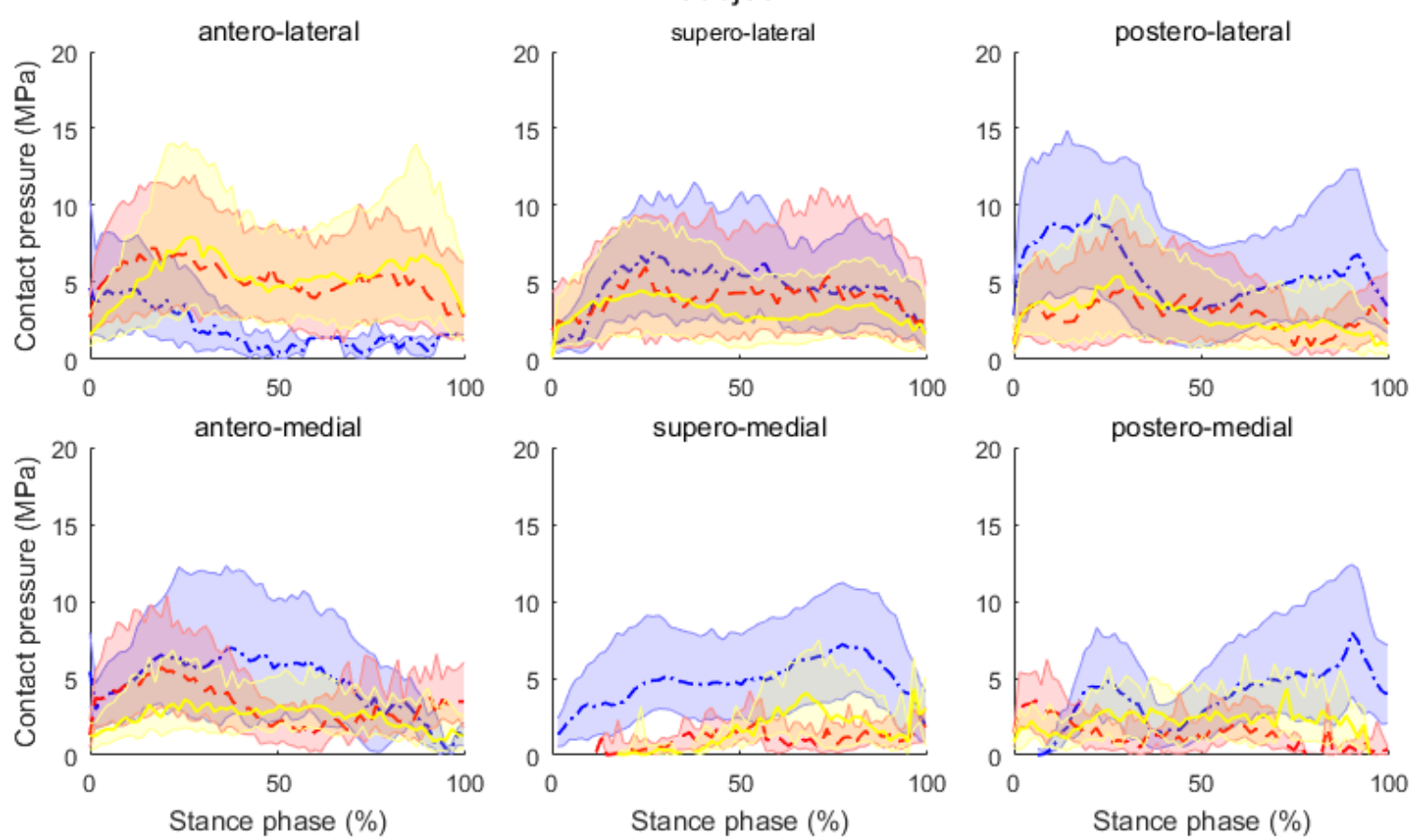

-.--.-..'Generic mesh $----\cdot$ Genericloadir

Subject-specific

Subject 2
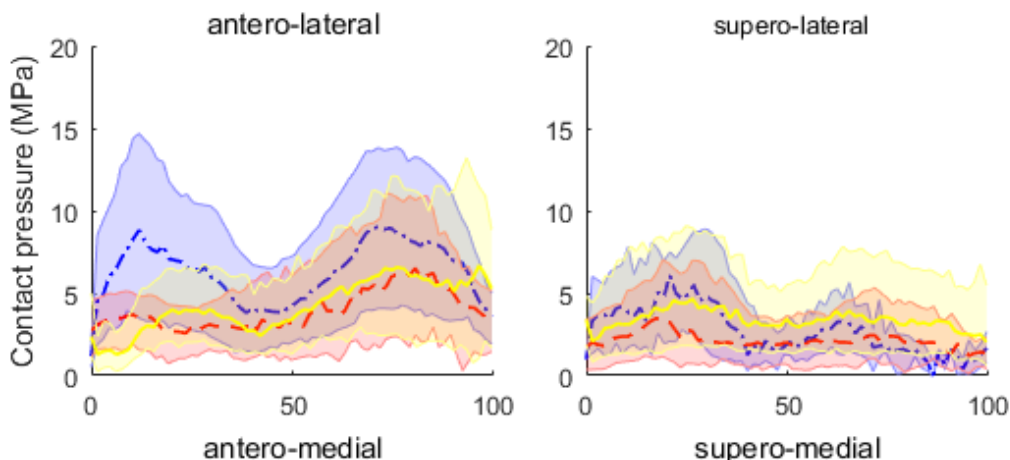

20 postero-lateral
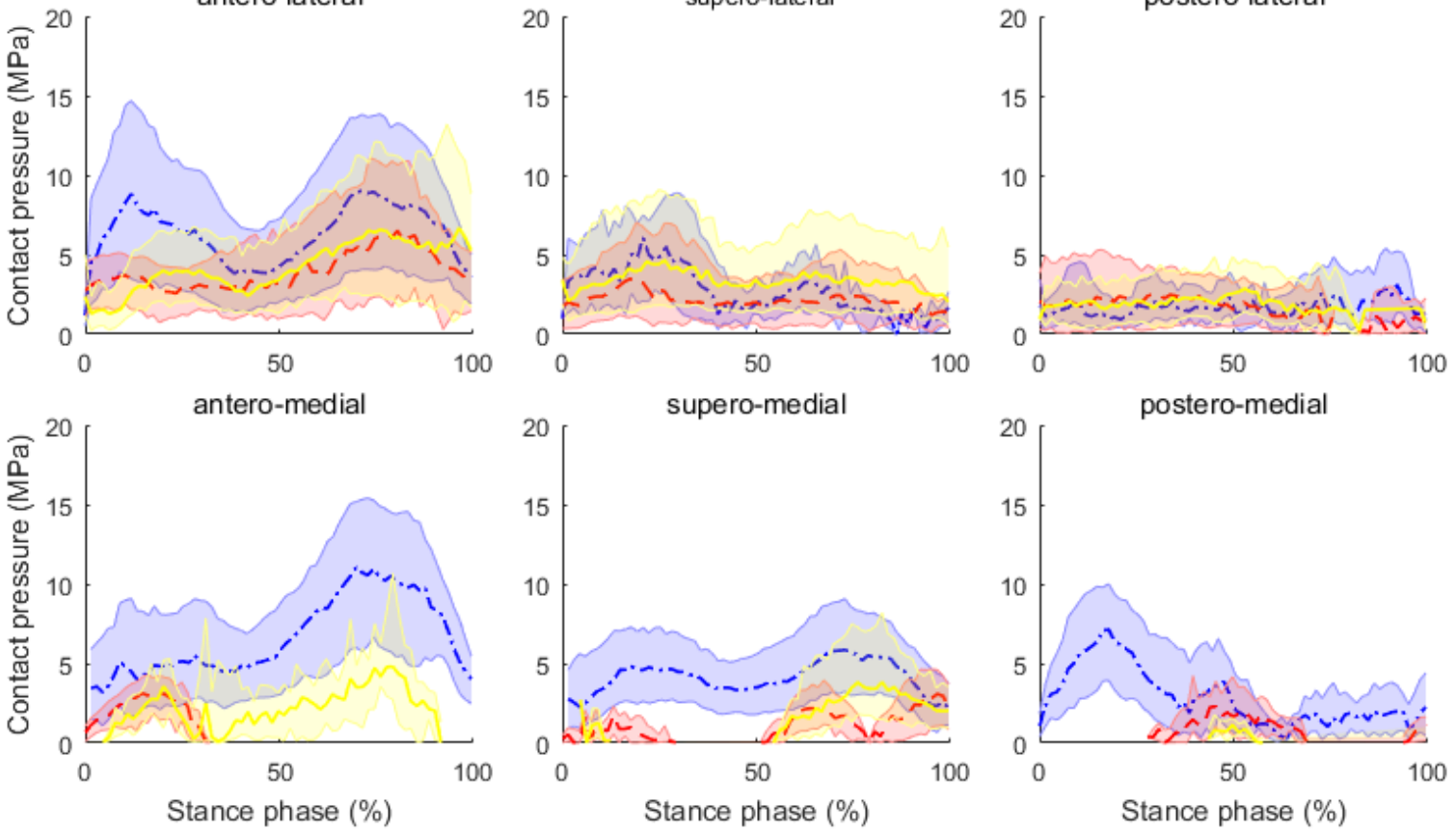

Figure 7. Median contact pressure (and $25^{\text {th }}$ and $75^{\text {th }}$ percentile) over stance phase for subject 1 and 2 using subject-specific mesh with subject-specific loading (yellow full line), subjectspecific mesh with generic loading (red dashed line) and generic mesh with subject-specific loading (blue dot-dashed line). 
Subject 1
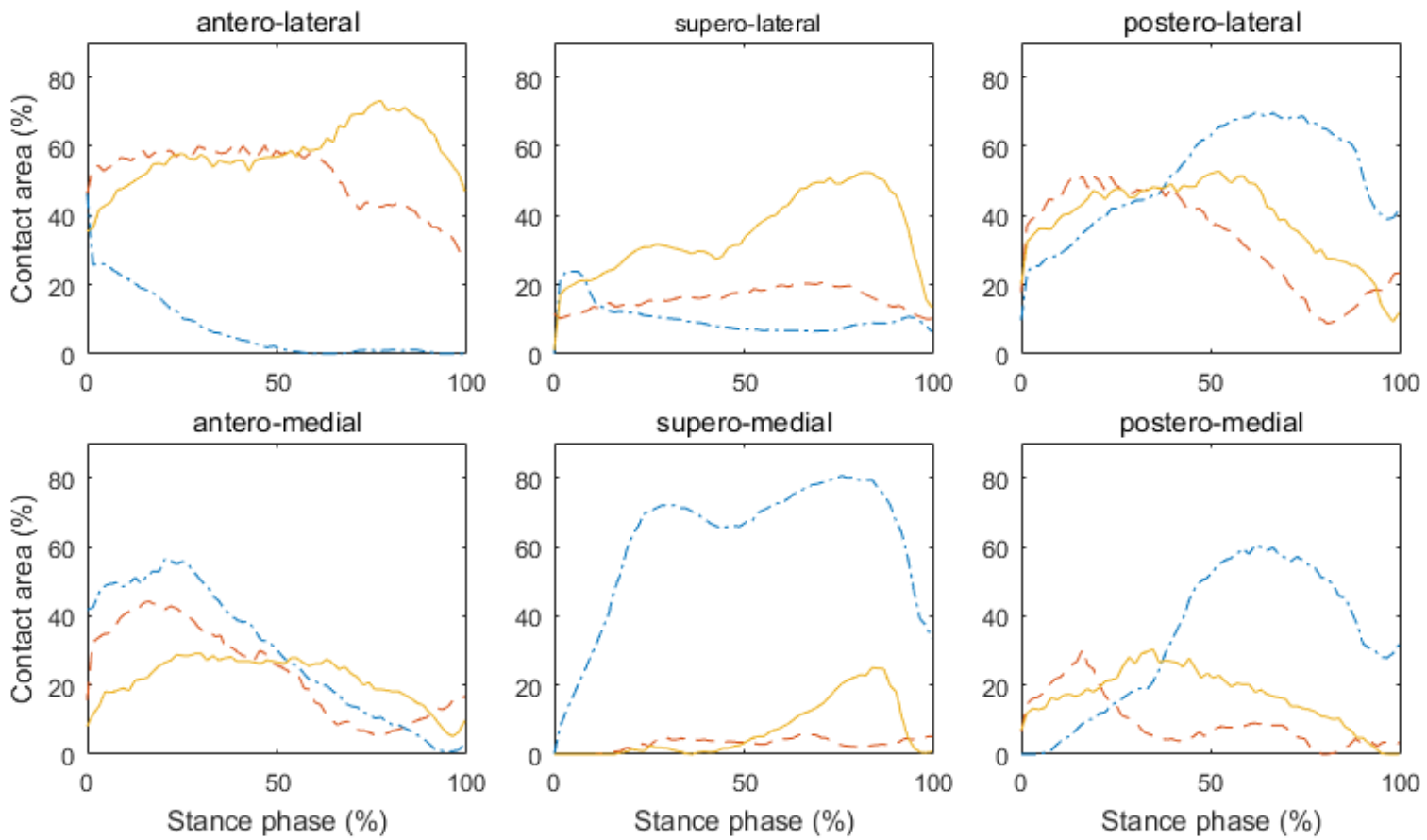

-.-.-. Generic mesh - - - - Generic loading Subject-specific
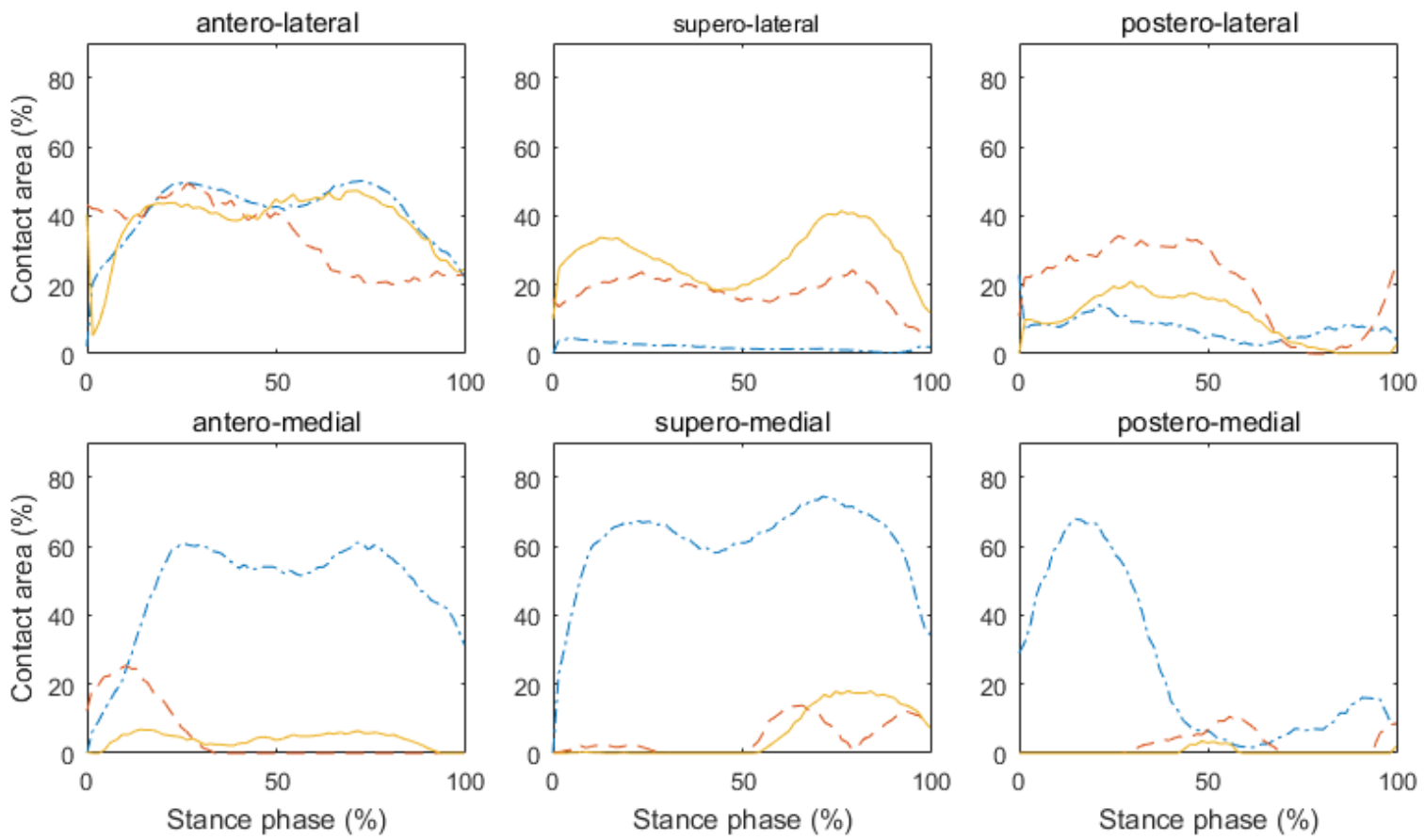

Figure 8. Contact area over stance phase for subject 1 and 2 using subject-specific mesh with subject-specific loading (yellow full line), subject-specific mesh with generic loading (red dashed line) and generic mesh with subject-specific loading (blue dot-dashed line). 